\title{
Mild COVID-19 infection in an NMO patient treated with tocilizumab: a confirmation of anti-IL- 6 protective role?
}

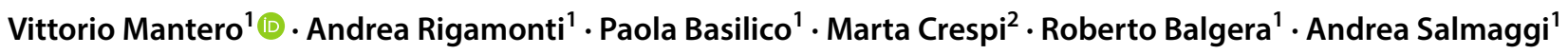

Received: 22 June 2020 / Revised: 25 June 2020 / Accepted: 30 June 2020 / Published online: 7 July 2020

(c) Springer-Verlag GmbH Germany, part of Springer Nature 2020

\section{Dear Editor,}

The novel coronavirus SARS-CoV-2 (which results in COVID-19) was recently discovered in Wuhan, China. Its morbidity and mortality have been associated with acute respiratory distress syndrome (ARDS) and consequent respiratory failure, with complications related to elderly age and comorbidities [1]. It is unclear if immunosuppressant treatment increases the risk of severe COVID-19, although preliminary data in patients with systemic autoimmune disorders suggest that chronic steroid treatment may not be associated with a higher risk of COVID-19 infection [2].

COVID-19 may primarily involve T lymphocytes, particularly diminishing CD8 $+\mathrm{T}$ and CD4 $+\mathrm{T}$ cells [1]; some patients with severe COVID-19 may experience a cytokine storm syndrome as a hyperinflammatory response to the virus [3]. Interleukin (IL)-6 is one of the principal cytokines involved in infection-induced cytokine storm, therefore treatment of cytokine storm induced by COVID-19 with tocilizumab (an anti-IL-6 monoclonal antibody) has been proposed and in China tocilizumab is recommended as a therapy for critical patients.

Herein, we present the case of a 44-year-old neuromyelitis optica (NMO) physician woman treated with tocilizumab, developing a mild COVID-19 infection without sequelae.

The patient had a history of generalized myasthenia gravis in 2010, with positivity for anti-acetylcholine receptor antibodies, treated with VATET after 6 months with the detection of thymic hyperplasia and subsequent clinical remission. She was a smoker (10 cigarettes daily from 10 years) and had a BMI of 19.3. In October 2016, she was

Vittorio Mantero

vittorio.mantero@hotmail.com

1 MS Center, Department of Neurology, ASST Lecco, Via Dell’Eremo 9/11, 23900 Lecco, Italy

2 Neurosurgery Unit, “A. Manzoni” Hospital - ASST Lecco, Lecco, Italy hospitalized for dorsal myelitis D2-D4 with positivity for anti-aquaporin4 antibodies. In October 2018 she presented a relapse with myelitis D4-D5, for which rituximab therapy was started and continued until October 2019, when she presented a new relapse with extension of the dorsal myelitis. On November 8th 2019 therapy with tocilizimub at the dosage of $8 \mathrm{mg} / \mathrm{kg}$ every 28 days was started. The last doses of tocilizumab were administered on February 27th, March 26th and April 23th, 2020. At the time of the last infusion, patient had B-cell depletion (2 CD19+, 2 CD20+ and $0.1 \mathrm{CD} 27+$ cell $/ \mathrm{mm}^{3}$ ) at peripheral blood lymphocyte immunophenotype. Expanded disability status scale was 1.5 (suspended hypoaesthesia/dysaesthesia below the right mammillary line and deep tendon reflexes).

On May 5th she developed nausea, which worsened the following day with the appearance of foul-smelling diarrhea and intense headache. On May 7th and 8th, she presented low-grade fever $\left(37^{\circ} \mathrm{C}\right)$ and abdominal pain always associated with nausea and headache. Moreover, a pseudo-relapse with worsening of paresthesias in the lower limbs occurred. Because of her job as a hospital doctor, she had been in close contact with many positive patients during the epidemics. A nasopharyngeal swab was negative for SARS-CoV-2 in a real-time reverse transcriptase-polymerase chain reaction assay. Chest CT was negative. Blood exam showed normal leucocyte and lymphocyte count and C-reactive protein levels. Subsequent serological testing revealed the presence of IgG and IgM for COVID-19. On May 21th, tocilizumab administration was performed as of scheduled treatment.

In this report, we describe the first case of an anti-IL-6 treated patient that developed SARS-COV-2 infection, without serious complications. Moreover, our patient had previously been treated with an anti-CD20 monoclonal antibody (about 7 months before the infection) and presented B-cell depletion. Only an NMO patient treated with rituximab who developed mild respiratory symptoms with COVID-19 was reported before [4]. 
A randomized, open-label, head-to-head study comparing intravenous tocilizumab versus azathioprine showed that tocilizumab significantly reduced relapses and stabilized NMO spectrum disease (NMOSD) patients [5]. To date, there is no recommendation to stop treatments used in NMOSD patients during COVID-19 pandemic [6]. In fact, relapses in patients with NMOSD may be devastating, and patients should be encouraged to continue therapies for attack prevention [7].

Cytokine storm is an important factor in the rapid progression of COVID-19. Since IL-6 is also considered to be a key mediator of cytokine release syndrome (CRS), drugs that inhibit IL-6 as tocilizumab can block CRS, playing a role in the treatment of cytokine storm caused by COVID19 [8]. The usage of tocilizumab for the treatment of CRS has been approved by the US FDA and is now in undergoing formal testing clinical trials. Preliminary data of case series show that tocilizumab could be an effective treatment to reduce mortality in patients with SARS-COV-2 infections [9-12].

We hypothesized that the previous use of anti-IL-6 may have played a protective role in this patient, avoiding the aggravation of symptoms. Our case might indicate that patients treated with tocilizumab or other anti-IL-6 antibodies, could be at lower risk from severe complications of COVID-19. This is a single observation, so definite conclusions cannot be drawn. There are international registries that are attempting to capture data on severity and recovery from COVID-19 in patients with multiple sclerosis and NMO according to various ongoing immunomodulatory/ immunosuppressive treatments [13]. So far, the preliminary available evidence suggests that immunosuppressive therapy does not seem to be associated with increased risk/ severity of COVID-19 infections [14], although data are somehow conflicting [15]. Further epidemiological studies are needed to assess the putative changes in the risk of contracting SARS-COV-2 infections in patients chronically treated with tocilizumab.

Author contributions VM contributed to the conceptualization, gathering of data and drafting the manuscript; AR, PB, MC, and RB contributed to gathering of data and drafting the manuscript; AS contributed to drafting and revising the manuscript.

\section{Compliance with ethical standards}

Conflicts of interest The authors declare that they have no competing financial interests or personal relationships that could have appeared to influence the work reported in this paper.

Ethical approval All procedures performed in this study were in accordance with the ethical standards of the institutional and national research committee and with the 1964 Helsinki declaration and its later amendments or comparable ethical standards.
Informed consent The patient provided consent to be anonymously included in this report.

\section{References}

1. Chen G, Wu D, Guo W et al (2020) Clinical and immunologic features in severe and moderate coronavirus disease 2019. J Clin Invest 130:2620-2629. https://doi.org/10.1172/JCI137244

2. Emmi G, Bettiol A, Mattioli I et al (2020) SARS-CoV-2 infection among patients with systemic autimmune diseases. Autoimmun Rev 19:102575. https://doi.org/10.1016/j.autrev.2020.102575

3. Mehta P, McAuley DF, Brown M et al (2020) COVID-19: consider cytokine storm syndromes and immunosuppression. Lancet 395:1033-1034. https://doi.org/10.1016/S0140-6736(20)30628-0

4. Creed MA, Ballesteros E Jr, Imitola J (2020) Mild COVID-19 infection despite chronic $\mathrm{B}$ cell depletion in a patient with aquaporin4-positive neuromyelitis optica spectrum disorder. Mult Scler Relat Disord 44:102199. https://doi.org/10.1016/j.msard.2020.102199

5. Zhang C, Zhang M, Qiu W et al (2020) Safety and efficacy of tocilizumab versus azathioprine in highly relapsing neuromyelitis optica spectrum disorder (TANGO): an open-label, multicentre, randomised, phase 2 trial. Lancet Neurol 19:391-401. https://doi. org/10.1016/S1474-4422(20)30070-3

6. Carnero Contentti E, Correa J (2020) Immunosuppression during the COVID-19 pandemic in neuromyelitis optica spectrum disorders patients: a new challenge. Mult Scler Relat Disord 41:102097. https ://doi.org/10.1016/j.msard.2020.102097

7. Brownlee W, Bourdette D, Broadley S, Killestein J, Ciccarelli O (2020) Treating multiple sclerosis and neuromyelitis optica spectrum disorder during the COVID-19 pandemic. Neurology 94:949952. https://doi.org/10.1212/WNL.0000000000009507

8. Zhang S, Li L, Shen A, Chen Y, Qi Z (2020) Rational use of tocilizumab in the treatment of novel coronavirus pneumonia. Clinical Drug Investig 40:511-518. https://doi.org/10.1007/s40261-02000917-3

9. Xu X, Han M, Li T, Sun W, Wang D, Fu B, Zhou Y, Zheng X, Yang Y, Li X, Zhang X, Pan A, Wei H (2020) Effective treatment of severe COVID-19 patients with tocilizumab. Proc Natl Acad Sci USA 117(20):10970-10975. https://doi.org/10.1073/pnas.20056 15117

10. Luo P, Liu Y, Qiu L, Liu X, Liu D, Li J (2020) Tocilizumab treatment in COVID-19: a single center experience. J Med Virol 92:814 818. https://doi.org/10.1002/jmv.25801

11. Alattar R, Ibrahim TBH, Shaar SH, Abdalla S, Shukri K, Daghfal JN et al (2020) Tocilizumab for the treatment of severe coronavirus disease 2019. J Med Virol. https://doi.org/10.1002/jmv.25964

12. Toniati P, Piva S, Cattalini M, Garrafa E, Regola F, Castelli F et al (2020) Tocilizumab for the treatment of severe COVID-19 pneumonia with hyperinflammatory syndrome and acute respiratory failure: a single center study of 100 patients in Brescia, Italy. Autoimmun Rev 19:102568. https://doi.org/10.1016/j.autrev.2020.102568

13. Sormani MP (2020) An Italian programme for COVID-19 infection in multiple sclerosis. Lancet Neurol. https://doi.org/10.1016/S1474 $-4422(20) 30147-2$

14. Novi G, Mikulska M, Briano F et al (2020) COVID-19 in a MS patient treated with ocrelizumab: does immunosuppression have a protective role? Mult Scler Relat Disord 42:102120. https://doi. org/10.1016/j.msard.2020.102120

15. Safavi F, Nourbakhsh B, Azimi AR (2020) B-cell depleting therapies may affect susceptibility to acute respiratory illness among patients with multiple sclerosis during the early COVID-19 epidemic in Iran. Mult Scler Relat Disord 43:102195. https://doi.org/10.1016/j.msard .2020 .102195 